\title{
Spiking Phineas Gage: A Neurocomputational Theory of Cognitive-Affective Integration in Decision Making
}

\author{
Brandon M. Wagar and Paul Thagard \\ University of Waterloo
}

\begin{abstract}
The authors present a neurological theory of how cognitive information and emotional information are integrated in the nucleus accumbens during effective decision making. They describe how the nucleus accumbens acts as a gateway to integrate cognitive information from the ventromedial prefrontal cortex and the hippocampus with emotional information from the amygdala. The authors have modeled this integration by a network of spiking artificial neurons organized into separate areas and used this computational model to simulate 2 kinds of cognitive-affective integration. The model simulates successful performance by people with normal cognitive-affective integration. The model also simulates the historical case of Phineas Gage as well as subsequent patients whose ability to make decisions became impeded by damage to the ventromedial prefrontal cortex.
\end{abstract}

Some people like to make decisions by flipping a coin, after assigning one choice to heads and another to tails. The point is not to make the decision indicated by the flip but rather to see how they feel about the choice that the coin flip tells them to do. Flipping the coin is an effective way to find out their emotional reactions to various alternatives, indicating the emotional weight they attach to them. From the perspective of mathematical theories of decision making such as those that say that people do or should maximize expected utility, the coin-flip exercise is bizarre. But, there is increasing appreciation in cognitive science that emotions are an integral part of decision making (e.g., Churchland, 1996; A. Damasio, 1994; Finucane, Alhakami, Slovic, \& Johnson, 2000; Lerner \& Keltner, 2000, 2001; Loewenstein, Weber, Hsee, \& Welch, 2001; Rottenstreich \& Hsee, 2001). In this article, we present a neurocomputational theory of how the brain produces these covert emotional reactions.

Current artificial neural network models of cognitive-affective processing use simplified neurons and neglect neuroanatomical information about how different parts of the brain contribute to decision making (Mischel \& Shoda, 1995; Nerb \& Spada, 2001; Thagard, 2000, 2003). Here, we present a new computational model, GAGE, which is much more neurologically realistic than previous models. It organizes neurons into anatomically recognized groups corresponding to crucial brain areas, including the ventromedial prefrontal cortex (VMPFC), the hippocampus, and the amygdala. Our model shows how another area, the nucleus

Brandon M. Wagar, Department of Psychology, University of Waterloo, Waterloo, Ontario, Canada; Paul Thagard, Department of Philosophy, University of Waterloo.

This research was supported by the Natural Sciences and Research Council of Canada. The authors wish to thank Barbara Bulman-Fleming, Mike Dixon, Chris Eliasmith, Josef Nerb, Steve Smith, and Chris White for useful comments on drafts of the manuscript.

Correspondence concerning this article should be addressed to Brandon M. Wagar, Department of Psychology, University of Waterloo, 200 University Avenue West, Waterloo, Ontario N2L 3G1, Canada. E-mail: bmwagar@uwaterloo.ca accumbens (NAcc), can serve to integrate cognitive information from the VMPFC and the hippocampus with emotional information from the amygdala. Consistent with A. Damasio's (1994) somatic-marker hypothesis, our model indicates that the VMPFC and the amygdala interact to produce emotional signals (i.e., somatic markers) indicating expected outcomes and that these expected outcomes compete with immediate outcomes for amygdala output. However, our model moves beyond A. Damasio's claim, highlighting the importance of the NAcc gateway. For the somatic markers from the VMPFC and the amygdala to access those brain areas responsible for higher order reasoning, context information from the hippocampus must unlock the NAcc gate, allowing this information to pass through. Also, the individual neurons in our model are more realistic than those used in most artificial neural network models in cognitive science because they exhibit the spiking behavior found in real neurons. This level of representation highlights another important aspect of cognitive-affective integration in the brain: time. GAGE shows that temporal coordination between the VMPFC and the amygdala is a key component in eliciting covert emotional reactions to stimuli.

Our model is implemented by a computer program that successfully simulates two kinds of cognitive-affective integration: people's performance on the Iowa gambling task (Bechara, Damasio, Damasio, \& Anderson, 1994) and the integration of physiological arousal and cognition in determining emotional states (Schachter \& Singer, 1962). In the Iowa gambling task, people with intact brains are able to use covert emotional reactions to guide their behavior when choosing cards, whereas people with damage to the VMPFC are unable to use such reactions to play the game successfully. According to Bechara et al. (1994), this deficit is the result of their inability to integrate cognitive and emotional information to discern the future consequences of their actions. In people with intact brains, it is the emotional reactions to good and bad cards that signal predicted outcomes and guide people's behavior (cf. the coin-flip exercise). Our computational model shows both how people with intact brains perform cognitive-affective integration to succeed in the Iowa gambling task and why this integration breaks down in people with damage to the VMPFC. 
In addition to modeling performance on the Iowa gambling task, GAGE also models the integration of physiological arousal and cognition in determining emotional states (Schachter \& Singer, 1962). Schachter and Singer (1962) showed that given a state of emotional arousal for which no immediately appropriate explanation is available (i.e., ambiguous emotional arousal), participants could be manipulated into states of euphoria or anger by manipulating the context. All participants were injected with epinephrine, leading to the same state of physiological arousal among participants, yet this arousal led to different appraisals of emotional reaction as determined by the current context. Although we are not concerned with the high-level mechanism(s) underlying the cognitive appraisal of one's emotional state, we are interested in the mechanism whereby context exerts a moderating effect on the emotional reaction to different stimuli. Our computational model shows how context is capable of exerting such an effect.

The most famous person with damage to the VMPFC was a 19th-century patient, Phineas Gage. ${ }^{1}$ Our model, called GAGE in his honor, explains why his ability to make decisions declined drastically after he sustained brain damage presumed to have occurred in the VMPFC (H. Damasio, Grabowski, Frank, Galburda, \& Damasio, 1994). We therefore begin by reviewing his case and the somatic-marker hypothesis that A. Damasio (1994) developed to account for it. Our model is compatible with A. Damasio's explanation of the decision-making deficits of VMPFC damage but goes beyond it in providing a much more detailed and computationally implemented account of the role the NAcc plays in effective and defective decision making.

Phineas Gage was the foreman of a railway construction gang working for the contractors preparing the bed for the Rutland and Burlington railroad near Cavendish, VT. On September 13, 1848, an accidental explosion of a charge he had set blew his tamping iron through his head. Most of the front part of the left side of his brain was destroyed.

Some months after the accident, Phineas felt strong enough to resume work. But, because his personality had changed so much, the contractors who had employed him were reluctant to reinstate him. Before the accident, he had been their most capable and efficient foreman, one with a well-balanced mind and who was looked on as a shrewd, smart businessman. He was now fitful, irreverent, and grossly profane, showing little deference for his fellow coworkers. He was also unable to settle on any of the plans he devised for future action. His friends said he was "no longer Gage" (Macmillan, 2000).

\section{A. Damasio's (1994) Somatic-Marker Hypothesis}

Phineas Gage represents the first reported case of an individual who suffered damage to the VMPFC. What is of particular interest is the nature of the deficits resulting from damage to this brain region. Although basic cognitive, intellectual, and language skills remain intact, the ability to reason-particularly within a social context—is seriously impaired. Concretely, Gage could think and speak, yet he lost all of his friends and was unable to hold down a job. More important, Phineas Gage is not the only instance of this fascinating constellation of subtle deficits; there have been several other documented cases of lesions localized to the VMPFC (see A. Damasio, 1994). In general, VMPFC damage is characterized by insensitivity to future consequences. Although the impairment is usually discussed in the context of real-life decision making (i.e., as an impairment in the ability to predict the consequences of one's actions within a complex social environment), the impairment extends to other decision-making tasks that involve distinctions between long-term and short-term consequences in an environment containing punishment and reward. Evidently, damage to the VMPFC injures the ability to predict the future consequences of one's actions and behave accordingly.

According to Antonio Damasio (1994), the VMPFC is critically involved in the production of somatic markers. Somatic markers are the feelings, or emotional reactions, that have become associated through experience with the predicted long-term outcomes of certain responses to a given situation. According to the somaticmarker hypothesis, sensory representations of a given response to the current situation activate knowledge tied in with previous emotional experience. The resulting covert emotional signals (i.e., somatic markers) act as biases influencing the mechanisms responsible for higher level cognitive processes and/or the motor effector sites. Somatic markers assist people during the decision-making process by rapidly highlighting those options that have positive predicted outcomes and eliminating those options that have negative predicted outcomes from further consideration. Somatic markers make the decision process more efficient by narrowing the number of feasible behavioral alternatives while allowing the organism to reason according to the long-term predicted outcomes of its actions.

\section{Cognitive-Affective Integration in the NAcc}

We now present a neurological theory of how cognitive information and emotional information are integrated in the NAcc during effective decision making. A. Damasio's (1994) proposed somatic-marker mechanism suggests that interconnections between the VMPFC and the amygdala are responsible for the formation of memory traces that allow the organism to predict the future outcome of a given response. We expand on this mechanism (see Figure 1), discussing how somatic markers are passed on to the NAcc, which acts as a gateway allowing only contextconsistent behavior (as determined by hippocampal inputs to the NAcc) to pass through. In addition, GAGE shows that temporal coordination between the VMPFC and the amygdala is a key component to eliciting emotional reactions to stimuli. Information that passes through the NAcc is redirected back to the VMPFC and other prefrontal and neocortical sites, providing the covert emotional reaction that feeds into higher level cognitive processes and/or the motor effector sites.

In the following sections, we describe the processes of establishing predicted outcomes and gating of NAcc throughput in more detail. We then move to a description of the predicted large-scale

\footnotetext{
${ }^{1}$ It should be noted that the claim that Phineas Gage's brain damage included the VMPFC has recently been questioned (Macmillan, 2000). Given indeterminacy about where the tamping iron entered and left the skull, individual differences in the position of the brain inside the skull, and the fact that Harlow's (1848) accounts are questionable, we might never know the true nature of Gage's lesions. However, the fact remains that, regardless of these concerns, the folklore surrounding his story still makes Gage the most famous person with apparent damage to the VMPFC.
} 


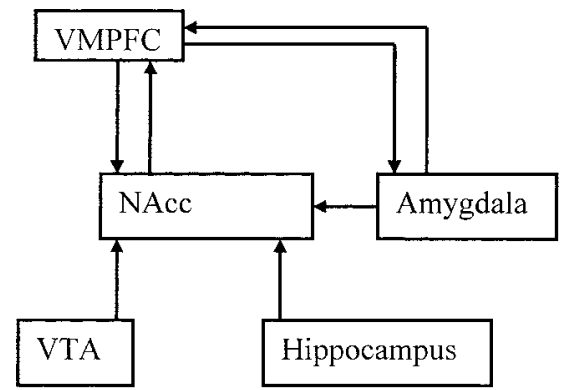

Figure 1. Schematic of the neuronal mechanism implemented in GAGE. $\mathrm{VMPFC}=$ ventromedial prefrontal cortex NAcc $=$ nucleus accumbens; $\mathrm{VTA}=$ ventral tegmental area.

dynamics of the mechanism using a test of VMPFC damage as an example.

\section{Establishing Predicted Outcomes}

Emotional signals in decision making bias the organism to avoid decisions that will lead to negative future outcomes and promote decisions that will lead to positive future outcomes. In this manner, the neural basis for the production of emotional signals impels the organism to behave in ways that promote achievement and longterm survival within a given environment. To this end, the mechanism must recruit brain regions involved in the processing and storage of bodily states (to avoid unpleasant states and promote homeostatic ones). It must also recruit regions responsible for cognitive processes (to process sensory representations), to be able to link the latter to the former.

A. Damasio (1994) highlighted two key structures as primarily responsible for the production of somatic markers: the VMPFC and the amygdala. The VMPFC receives input from sensory cortices, representing the behavioral options, and from limbic structures, most notably the amygdala, which processes somatic states. Through these interconnections between cognitive and emotional processes, the VMPFC records the signals that define a given response by encoding the representations of certain stimuli and the behavioral significance of the somatic states that have been previously associated with the given response. It thereby lays down a memory trace (somatic marker) that represents a given action and the expected consequences of the action. Once the memory trace is encoded, the VMPFC houses the critical output for somatic markers to influence decision making. When a set of inputs to the VMPFC elicit a response, the VMPFC, through its reciprocal connections with the amygdala, elicits a reenactment of the bodily state consistent with the predicted future outcome of the given behavior. Then, this covert emotional reaction is passed on to overt decision-making processes and/or motor effector sites. If the predicted outcome is positive, the response remains active for further consideration or is chosen for action. If the predicted outcome is negative, the option is eliminated from the set of possible alternatives.

\section{Gating of NAcc Throughput}

The mechanism responsible for the production of emotional signals in decision making pretunes the organism to behave in ways that promote achievement and long-term survival within a given environment. To this end, A. Damasio's (1994) somaticmarker mechanism elicits emotional reactions signaling the predicted outcome of a given event. We now build on this mechanism, demonstrating hippocampal gating of the prefrontal cortex throughput in NAcc neurons (see Figure 2 for a schematic depiction). Our extended mechanism describes how the NAcc narrows
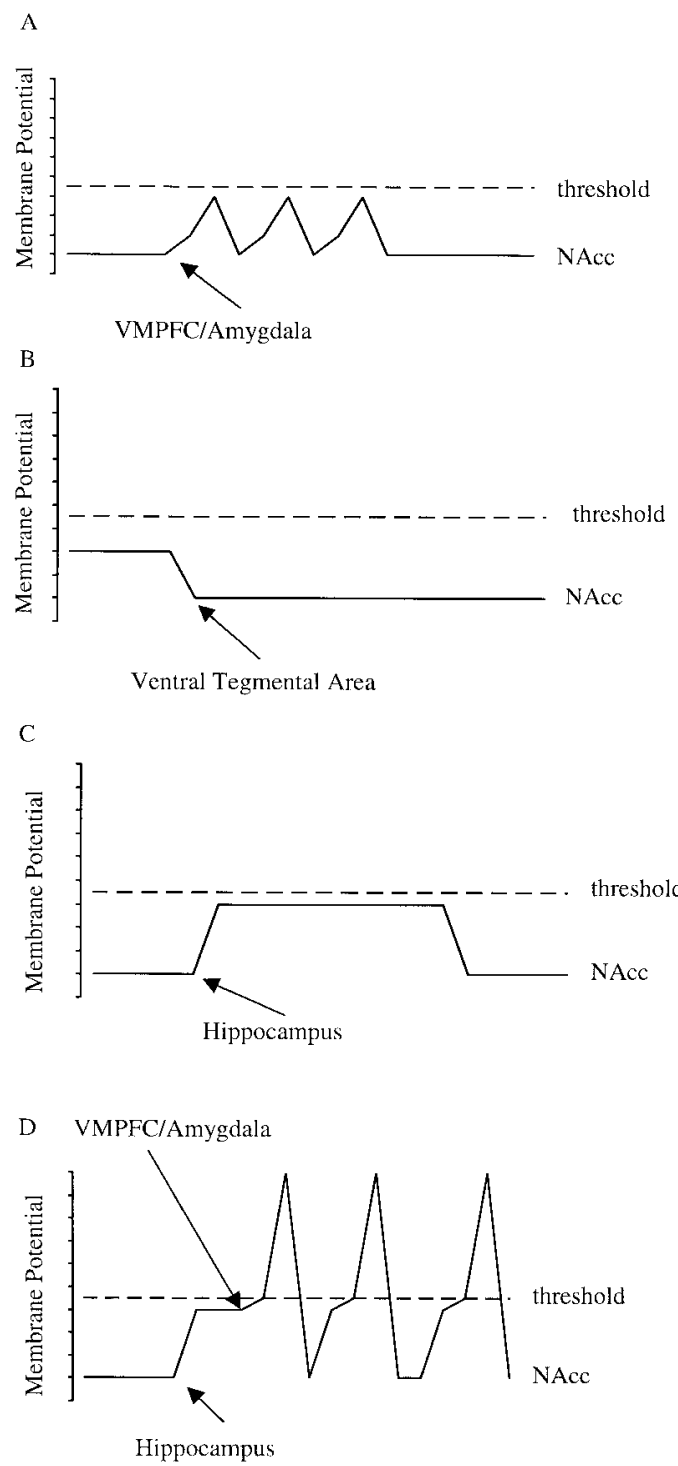

Figure 2. The nucleus accumbens (NAcc) gateway. A: The ventromedial prefrontal cortex (VMPFC) and the amygdala produce brief, smallamplitude membrane depolarizations in NAcc, which themselves cannot cause the NAcc neurons to fire. B: This is because NAcc neurons are typically in a hyperpolarized state as a result of the massive inhibitory dopamine input from the ventral tegmental area. C: Hippocampal input, conversely, produces large-amplitude, long-duration, plateaulike depolarizations. D: For the subset of NAcc neurons receiving hippocampal input, their typical hyperpolarized state is disrupted by temporary depolarization plateaus that bring NAcc neurons' activity levels close to firing threshold, thereby allowing any coincidental VMPFC and amygdala activity to elicit spike activity in NAcc neurons and pass through the NAcc gateway. 
down the alternative choices by allowing only those behaviors that are consistent with the current context to access higher level cognitive processes and/or the motor effector sites responsible for action.

Hippocampal input limits NAcc gateway throughput to those behaviors that are consistent with the current context (see Figure 3; O'Donnell \& Grace, 1995). The NAcc is responsible for mediating basic locomotor and appetitive behaviors that are driven by the affective state of the organism (Mogenson, Jones, \& Yim, 1980). Thus, it provides the ideal location for cognitive-affective integration during covert decision-making tasks. To this end, recent studies have shown that the NAcc is involved in reward learning (Berns, McClure, Pagnoni, \& Montague, 2001; Breiter, Aharon, Kahneman, Dale, \& Shizgal, 2001; Knutson, Adams, Fong, \& Hommer, 2001; Knutson, Westdorp, Kaiser, \& Hommer, 2000), drug addiction (Calabresi, De Murtas, \& Bernardi, 1997; Hitchcott \& Phillips, 1997; Mogenson et al., 1980), and affective processes (Calabresi et al., 1997; Mogenson et al., 1980).

The NAcc receives afferent connections from the VMPFC, the amygdala, and the hippocampus and massive dopamine input from the ventral tegmental area (see Figure 1; O'Donnell \& Grace, 1995). The VMPFC and the amygdala produce brief, smallamplitude membrane depolarizations in the NAcc, which themselves cannot cause NAcc neurons to fire (Grace \& Moore, 1998; O'Donnell, 1999; O’Donnell \& Grace, 1995). This is because NAcc neurons are typically in a hyperpolarized state as a result of the massive inhibitory dopamine input from the ventral tegmental area (Grace \& Moore, 1998; O’Donnell, 1999; O’Donnell \& Grace, 1995). Therefore, NAcc neurons are being constantly bombarded with VMPFC- and amygdala-driven excitatory postsynaptic potentials, yet none of the information is getting through. Hippocampal input, conversely, produces large-amplitude, long-

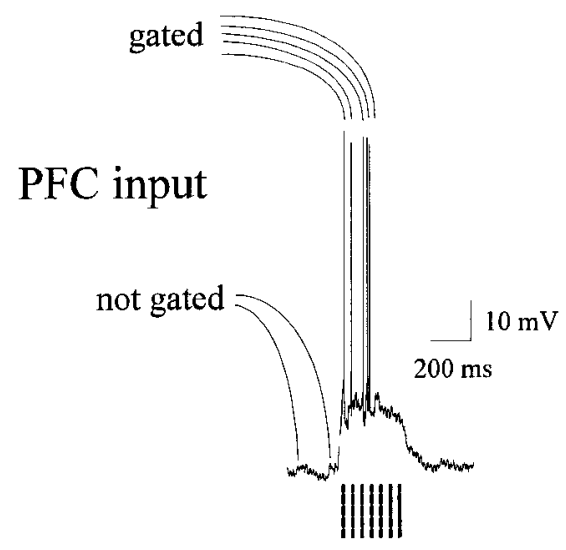

Figure 3. Hippocampal gating of the prefrontal cortex (PFC) throughput in nucleus accumbens (NAcc) neurons. During the down state, excitatory postsynaptic potentials (EPSPs) from the PFC afferent activation (indicated by the lines labeled not gated) do not result in NAcc firing. The up state is dependent on hippocampal inputs (indicated by the broken upward lines beneath the tracing). Because the up state elicited by hippocampal inputs brings the NAcc membrane potential close to firing threshold, EPSPs from the PFC afferent activation (indicated by the lines labeled gated) easily elicit NAcc firing. Adapted from "Ensemble Coding in the Nucleus Accumbens," by P. O’Donnell, 1999, Psychobiology, 27, p. 188. Copyright 1999 by the Psychonomic Society. Adapted with permission. duration, plateaulike depolarizations (Grace \& Moore, 1998; O'Donnell, 1999; O'Donnell \& Grace, 1995). For the subset of NAcc neurons receiving hippocampal input, their typical hyperpolarized state is disrupted by temporary depolarization plateaus that bring NAcc neurons' activity levels close to firing threshold, thereby allowing any coincidental VMPFC and amygdala activity to elicit spike activity in NAcc neurons and pass through the NAcc gateway (see Figure 3).

Thus, the hippocampus controls VMPFC and amygdala throughput in the NAcc by allowing only those patterns active in NAcc neurons that are consistent with the current context to elicit spike activity in NAcc neurons. The combined VMPFC and amygdala input to NAcc neurons represents the multitude of potentially effective responses (and their associated emotional reactions) to a given situation. The hippocampus influences the selection of a given response by facilitating within the NAcc only those responses that are congruent with the current context.

The amygdala provides a facilitatory influence on VMPFC activity in the NAcc, but only if the amygdala activity precedes VMPFC stimulation within a brief, 40-ms period (Grace \& Moore, 1998; O’Donnell \& Grace, 1995). This event-related facilitation provides the means by which an emotional valence representing the predicted outcome of a given response can be passed through the NAcc to higher level cognitive processes, thereby creating the covert emotional reaction predicting the future outcome of a given response. The strict time constraint here also highlights the importance of a coincidental increase in firing rate between the VMPFC and the amygdala in cognitive-affective integration. Because the VMPFC is constantly bombarding the NAcc with multiple patterns, the amygdala activity must be in close temporal proximity to the appropriate VMPFC response. If the amygdala input to NAcc neurons was to exert a long-lasting influence, emotional valences could become inappropriately associated with alternative responses.

Because the VMPFC elicits a predicted outcome through reciprocal connections with the amygdala, the VMPFC signal representing a given response and the amygdala signal representing the emotionally laden predicted outcome of that response are generated together and will arrive at NAcc neurons together. This allows them to fulfill the strict time constraints of the event-related facilitation previously mentioned. Also, because the amygdala produces rapid, small-amplitude excitatory postsynaptic potentials, the representation of a given response and its associated emotional valence will degrade rapidly, preventing the occurrence of any possible confusion between response and predicted outcome.

In summary, the NAcc and the hippocampus are key contributors to the covert production of emotional reactions, which can direct decision-making processes. The NAcc forms a gateway for somatic markers, and the hippocampus determines what passes through this gateway by limiting throughput to those responses that are consistent with the current environment.

\section{Network Dynamics}

Information representing the current context, bodily states associated with the current situation, and potential responses to and appraisals of the current situation are represented in the hippocampus, amygdala, and VMPFC, respectively (see Figure 4 for a schematic depiction). Through memories stored in its reciprocal 
A

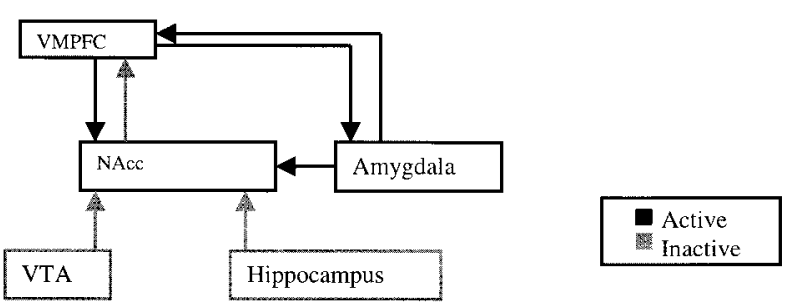

$\mathrm{B}$

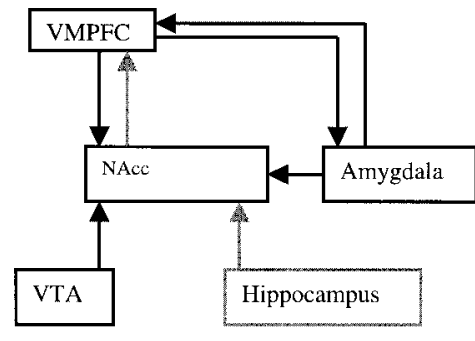

$\mathrm{C}$

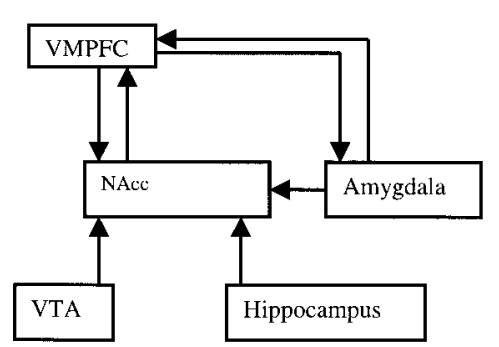

$\mathrm{D}$

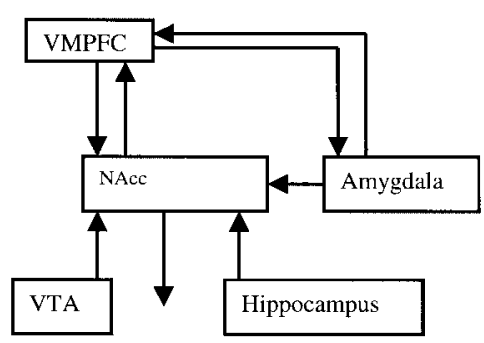

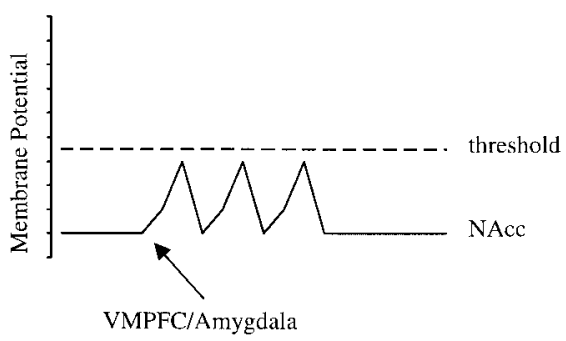

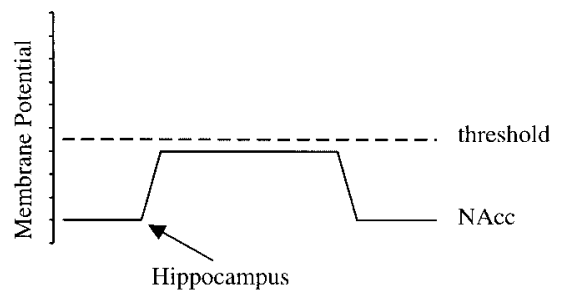

VMPFC/Amygdala

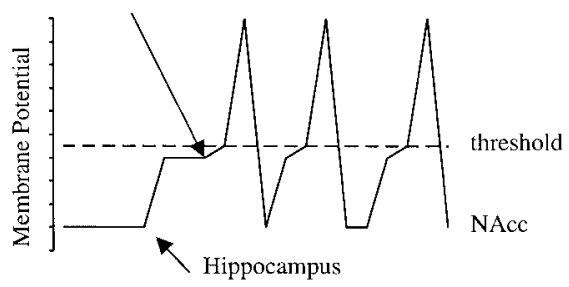

Figure 4. Network dynamics. A: Through memories stored in its reciprocal connections with the amygdala, the ventromedial prefrontal cortex (VMPFC) elicits an emotional signal representing the predicted future outcome of a given response, and this information is fed into the nucleus accumbens (NAcc). B: Because NAcc neurons are typically in a hyperpolarized state as a result of dopamine inhibition from the ventral tegmental area (VTA), the VMPFC and amygdala inputs alone are insufficient to elicit spike activity in NAcc neurons. C: However, a subset of NAcc neurons will become depolarized by hippocampal input representing the current context. D: Those responses in the VMPFC that are consistent with the current context (i.e., make synapses on NAcc neurons that are experiencing hippocampal depolarization) will then be able to elicit spike activity in NAcc neurons, thereby passing the given response and its emotionally laden predicted outcome on to higher level cognitive processes and/or motor effector sites.

connections with the amygdala, the VMPFC elicits an emotional signal representing the predicted future outcome of a given response, and this information is fed into the NAcc. Because NAcc neurons are typically in a hyperpolarized state as a result of dopamine inhibition from the ventral tegmental area, VMPFC and amygdala inputs alone are insufficient to elicit spike activity in NAcc neurons. However, a subset of NAcc neurons will become depolarized by hippocampal input representing the current context. Those responses in the VMPFC that are consistent with the current context (i.e., make synapses on NAcc neurons that are experiencing hippocampal depolarization) will then be able to elicit spike activity in NAcc neurons, thereby passing the given response and its emotionally laden predicted outcome on to higher level cognitive processes and/or motor effector sites. 
We have tested the proposed mechanism using a computer simulation that implements it in a network of spiking artificial neurons, GAGE. A critical component of the model is that input from the hippocampus is required to elicit NAcc firing. To ensure that this constraint was met, we compared in vivo electrophysiological recordings from rats (Grace \& Moore, 1998) and GAGE (see Figure 5). In the intact system, the up state elicited by hippocampal inputs depolarizes the NAcc membrane potential, enabling it to elicit NAcc firing. When hippocampal input is inactivated (either by a local anesthetic or lesioning GAGE), the NAcc membrane potential remains hyperpolarized, thereby preventing NAcc firing.
We have applied GAGE to simulations of two kinds of cognitive-affective integration: people's performance on the Iowa gambling task and the integration of physiological arousal and cognition in determining emotional states. In Experiment 1, we hypothesized that the network would elicit emotional reactions (signifying which card to choose from a deck) based on the predicted outcome of a given response, even though the immediate outcome contradicts the future outcome. We specifically found that-consistent with the performance of human patients—-when presented with a given response, an intact network functions in this way, whereas a network in which the VMPFC has been lesioned makes decisions based on immediate outcomes rather than future outcomes.

A

hippocampal input intact

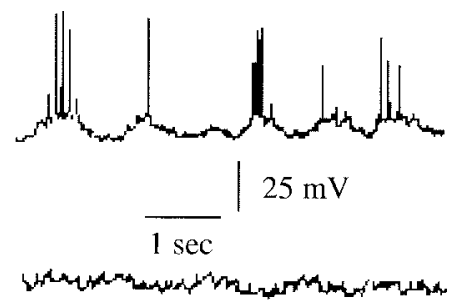

B

hippocampal input inactivated
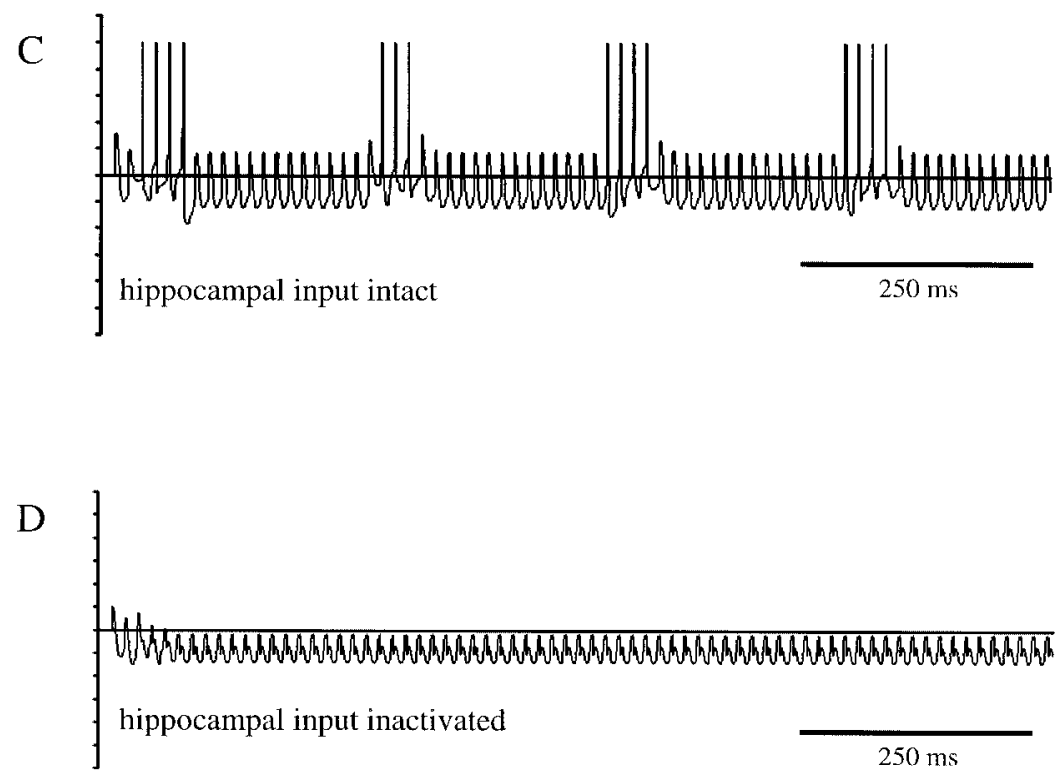

Figure 5. Input from the hippocampus is required to elicit nucleus accumbens (NAcc) firing: Comparison of in vivo electrophysiological recording from rats (A and B) and GAGE (C and D). A: In the intact system, the up state elicited by hippocampal inputs depolarizes the NAcc membrane potential, enabling it to elicit NAcc firing. B: When hippocampal input is inactivated by a local anesthetic, the NAcc membrane potential remains hyperpolarized, thereby preventing NAcc firing. C: In the intact network, the up state elicited by hippocampal inputs depolarizes the NAcc membrane potential, enabling it to elicit NAcc firing. D: When hippocampal input is removed, the NAcc membrane potential remains hyperpolarized, thereby preventing NAcc firing. A and B are adapted from "Regulation of Information Flow in the Nucleus Accumbens: A Model for the Pathophysiology of Schizophrenia," by A. Grace and H. Moore, in Origins and Development of Schizophrenia: Advances in Experimental Psychopathology (p. 134), 1998. Copyright 1998 by the American Psychological Association. Adapted with permission of the author. 
In Experiment 2, we hypothesized that in the presence of ambiguous emotional input, the network would elicit different emotional reactions depending on the current context. We specifically found that-consistent with the performance of human patientswhen presented with an emotional input for which no immediately appropriate explanation is available, NAcc throughput is determined by the context information from the hippocampus. That is, hippocampal input elicited different emotional reactions to a given physiological state, on the basis of the current context.

\section{GAGE}

In this section, we describe a network of spiking neurons, GAGE, based on the neural mechanism for cognitive-affective integration in the NAcc described above (see also Wagar \& Thagard, 2003). Figure 1 shows the overall architecture of the model. The model has 700 spiking neurons with 670 connections. The modeled regions consist of the VMPFC, the amygdala, the NAcc, the hippocampus, and the ventral tegmental area. Each region contains 100 neurons that receive input from other regions and/or external input and pass information on to other regions as well as 40 inhibitory interneurons.

The pattern of afferent, efferent, and internal connectivity follows that of the proposed neural mechanism. The model includes intraregional connections and interregional connections. Individual neurons were modeled as single-compartment integrate-andfire units, and synaptic interactions followed a Hebbian-based learning rule.

In the following section, we describe the layout of the various connection pathways. The equations for the neuronal and synaptic learning properties are summarized in the Appendix.

In constructing the model, we put emphasis on the incorporation of realistic network properties for the relative proportions of the various sets of connections composing the inter- and intraregional circuitry. Specific patterns of connectivity were classified as either sparse or dense on the basis of anatomical data. The sparse connection pattern resulted in each presynaptic neuron forming synapses with $30 \%$ of the neurons in the postsynaptic population. The dense connection pattern resulted in each presynaptic neuron forming synapses with $60 \%$ of the neurons in the postsynaptic population. Synaptic connections were distributed evenly over the postsynaptic population, with the pattern of connectivity determined in a random fashion. All connection strengths were initialized to a random value.

\section{Interregional Connectivity}

As can be seen from Figure 1, the NAcc receives afferent connections from all four of the remaining regions, each of which forms a dense connectivity pattern with NAcc transmission neurons (Calabresi et al., 1997; Mogenson et al., 1980). The VMPFC receives afferent connections from the NAcc and the amygdala, with the former establishing a dense connectivity pattern (Calabresi et al., 1997; Mogenson et al., 1980) while the latter establishes a sparse connectivity pattern (Aggleton, 2000). The amygdala receives afferent connections from the VMPFC, which are also sparse (Aggleton, 2000).

\section{Intraregional Connections}

Each of the modeled brain regions in the network contains inhibitory interneurons modeling the presence of basket cells in these neuronal populations. Basket cells project predominantly to the same layer where their soma are located and execute fastacting inhibitory postsynaptic potentials. In the model, basketlike inhibitory interneurons receive and project dense connections within each region.

Also modeled in our network are the NAcc gap junctions responsible for the lateral transfer of information among NAcc transmission neurons (O'Donnell, 1999). These gap junctions were modeled as having very sparse $(5 \%)$ connectivity between each of the transmission neurons in the NAcc population.

\section{Simulations}

All simulations were run using a program written in Java. Numerical integration of the differential equations for the membrane potentials and synaptic weight was performed with a time step of approximately $0.5 \mathrm{~ms}$. The generation of noise and statistical distribution of model parameters were based on Java's random-number-generator routines. The simulations were performed on a PC equipped with 640 megabytes of memory and a 750-MHz processor.

\section{Experiment 1}

The goal of Experiment 1 was to test GAGE's ability to simulate experimental results concerning the Iowa gambling task (Bechara et al., 1994). In this task, individuals are given a $\$ 2,000$ loan of play money and four decks of cards and instructed to make a series of card selections from any of the four decks until they are told to stop. Participants are instructed to play so that they maximize profit on the loan. Turning each card carries an immediate reward (which is large in Decks A and B and small in Decks C and D). Additionally, after turning some cards, the participants receive both a reward and a punishment (which is large in Decks A and B and small in Decks C and D). Playing mostly from the bad decks (A and B) results in an overall loss. Playing from the good decks $(\mathrm{C}$ and $\mathrm{D})$ results in an overall profit. Decks $\mathrm{A}$ and $\mathrm{B}$ are equivalent in terms of overall net loss, but in Deck A the punishment is more frequent and of smaller magnitude than in Deck B. Decks C and D are equivalent in terms of overall net gain, but in Deck $C$ the punishment is more frequent and of smaller magnitude than in Deck D. Participants are told that they are free to switch from any deck to another at any time and as often as they wish. However, participants are not told how many card selections must be made and are blind to the punishment schedules.

Typically, after encountering a few losses, normal participants adopt the strategy of picking predominantly from the good decks and avoiding the bad decks. This has been interpreted as indicating that normal participants are generating predicted outcomes in the form of covert emotional reactions (Bechara et al., 1994; Bechara, Damasio, Tranel, \& Damasio, 1997). However, patients with VMPFC damage are oblivious to the future consequences of their actions and pick predominantly from the bad decks. This is because such patients appear to be guided by immediate rewards (the high initial reward from the bad decks) rather than future outcomes 
(the overall profit from the good decks). The goal of Experiment 1 was to simulate the above described experimental results concerning the Iowa gambling task using GAGE.

\section{Method}

Stimuli. To simplify the experiment, we limited the stimuli to two decks rather than four. Because punishment schedule (e.g., the difference between Decks A and B) was not a predictive factor in the original experiments (see Bechara et al., 1994), we collapsed Decks A and B to form a single bad deck and Decks C and D to form a single good deck. On each trial in the experiment, GAGE was presented with a set of stimulus patterns consisting of activation vectors feeding into the VMPFC, the ventral tegmental area, the hippocampus, and the amygdala. Each pattern consisted of 50 active units (thereby exciting 50\% of the neurons in the receiving region). The VMPFC received either an activation pattern representing the good deck or an activation pattern representing the bad deck. The amygdala received an activation pattern representing a positive body state or an activation pattern representing a negative body state. For simplicity, each set of patterns (good deck-bad deck and positive-negative body state) formed complimentary pairs. Stimulation was achieved by applying constant synaptic excitation independently to each neuron corresponding to nodes active in the stimulus pattern.

Procedure. Initially, GAGE was trained on two combinations of stimuli: good deck with positive body state and bad deck with negative body state. This produced the requisite somatic markers between the VMPFC and the amygdala. Training occurred in an interleaved fashion over 4,000 time steps, with alternating stimulus combinations (positive body stategood deck and negative body state-bad deck) every 400 time steps. Hebbian learning was then turned off, and the model was presented with each of the test-stimulus combinations for epochs of 2,000 time steps to establish baseline ensemble activity in the NAcc for the expected representations.

Finally, the model was presented for epochs of 2,000 time steps with each of the test stimulus combinations. Test stimuli consisted of a given choice (e.g., good deck) and its immediate body state (e.g., negative body state). This allowed us to test whether the network would elicit emotional reactions based on future outcomes (e.g., pick the good deck even though the immediate outcome is negative) or immediate rewards (e.g., pick the bad deck even though the overall outcome is negative).

Hippocampal and ventral tegmental area inputs were constant throughout training, baseline, and test. The above experiments were performed with an intact network (simulating normal participants) and with a network in which the VMPFC had been removed after training (simulating patients with VMPFC damage). GAGE was lesioned by deleting all VMPFC connections.

Data collection and analysis. The spike trains (step-by-step record of whether a neuron was firing) for each neuron in the network were recorded for the entire run of the experiment. To characterize the ensemble activation pattern of the NAcc population (i.e., the subgroup of NAcc neurons that responded to each stimulus), we converted the spike trains into rate graphs. This was accomplished by placing a 20-time-step window over the spike train at each time step and taking the average number of spikes (i.e., the firing rate) within the window.

Once the rate graphs were acquired, cluster analysis was used to separate the entire NAcc population into those neurons that were active in response to the stimulus and those that were not responsive (see Everitt, Landau, \& Leese, 2001). In cluster analysis, a cluster is defined as a subset of elements that are cohesive among themselves but relatively isolated from the remaining elements. Cluster analysis sorts cases (e.g., neurons) into groups, or clusters, so that the degree of association is strong between members of the same cluster (e.g., neurons that are active) and weak between members of different clusters (e.g., neurons that are inactive). This was done for the baseline representations and the test representations.
The test representations were then compared with the baseline representations to determine the emotional reactions that GAGE passed through the NAcc in response to each of the decks. To determine GAGE's ability to elicit appropriate emotional reactions in response to test stimuli, we used signal-detection theory. In this analysis, $A^{\prime}$ was calculated (Snodgrass, Levy-Berger, \& Hayden, 1985). $A^{\prime}$ is a nonparametric analog to $d^{\prime}$, the signal-detection measure of sensitivity. To analyze the data, we calculated a hit rate, which is the proportion of active neurons in the baseline representation that were also active in the test representation, and a false alarm rate, which is the proportion of inactive neurons in the baseline representation that were also inactive in the test representation. This information was used to determine $A^{\prime}$ for each test representation. $A^{\prime}$ varies from 0 to 1 with .5 indicating chance performance. The larger $A^{\prime}$, the closer the test representation is to the baseline representation. The closer the test representation is to the baseline representation, the better is GAGE's ability to elicit appropriate emotional reactions in response to test stimuli.

\section{Results and Discussion}

To obtain a measure of GAGE's overall performance, we averaged all results over 50 replications of the experiment (with activation patterns and weights generated randomly for each replication). The results are presented in Figure 6.

The task of the network was to elicit a positive or negative emotional reaction given a choice (e.g., good deck) and an inconsistent body state (e.g., negative). That is, after having been trained on the predicted affective outcomes of each deck, the network was tested by presenting the VMPFC and the amygdala with activation patterns that simulated a condition in which future outcome and immediate outcome were in opposition. The goal was to determine whether an intact GAGE would elicit NAcc activation representing emotional reactions based on predicted future outcomes and

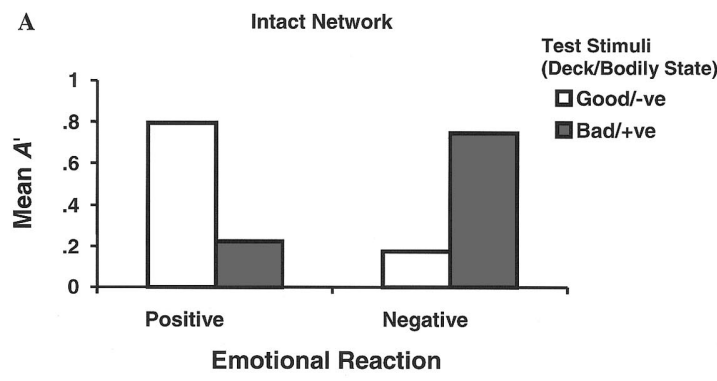

B

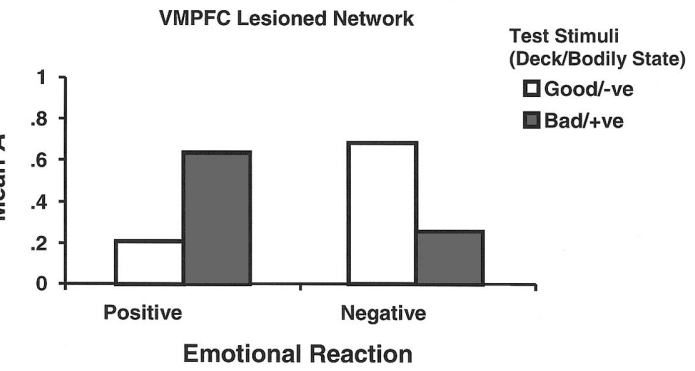

Figure 6. Mean $A^{\prime}$ (representing similarity to baseline) for test stimuli as a function of emotional reaction when GAGE is intact (A) and when the ventromedial prefrontal cortex (VMPFC) is lesioned in GAGE (B). - ve $=$ negative; $+\mathrm{ve}=$ positive 
whether the VMPFC-lesioned GAGE would elicit NAcc activation representing emotional reactions based on immediate outcomes.

As expected, the predicted affective outcome of a given response drives GAGE's behavior when the VMPFC is intact. Stored associations between the VMPFC and the amygdala are able to elicit a representation of the predicted future outcome of a given response. This information is then fed forward into the NAcc, and if it is consistent with the current context, it is passed on to higher level cognitive processes and/or the motor effector sites in the form of an emotional reaction.

Figure 6A shows the pattern of activity in NAcc neurons representing GAGE's ability to elicit appropriate emotional reactions in response to test stimuli. As can be seen, even in the presence of a conflicting emotional signal representing the immediate affective outcome of the response, GAGE elicits emotional reactions based on the predicted future outcome of the response. Assuming that the valence of these emotional reactions indicates which deck GAGE will choose from, the results show that when the VMPFC is intact, GAGE will predominantly choose from the good deck rather than the bad deck. It should be noted, however, that GAGE's performance was not all or none; there was a small percentage of occasions when it produced the complementary patterns of activity (eliciting a negative emotional reaction when presented with the good deck, $8 \%$; eliciting a positive emotional reaction when presented with the bad deck, $6 \%$ ). This is consistent with the performance of normal participants on the Iowa gambling task described above. Choosing from the good deck is the most efficient decision, as it ensures that an organism will behave in ways that promote long-term survival rather than short-term satisfaction. However, even normal participants take a risk sometimes and pick from the bad decks (Bechara et al., 1994).

Conversely, results from individuals suffering from VMPFC damage suggest that the mechanism no longer functions effectively. In these patients, immediate affective outcomes drive behavior rather than long-term benefits. Damage to the VMPFC prevents stored associations from eliciting a representation of the predicted future outcome of a given response. Thus, the only information fed into the NAcc is that which is initiated by the amygdala's response to current body states. Because the response promoted by this information is consistent with the current context (e.g., picking from the bad deck), the amygdala-driven decision is passed on to higher cognitive processes and/or the motor effector sites.

Figure 6B shows the pattern of activity in NAcc neurons representing GAGE's ability to elicit appropriate emotional reactions in response to test stimuli when the VMPFC has been lesioned. As can be seen, the immediate affective reward of a given response now drives behavior. GAGE now elicits emotional reactions based on the immediate outcome of the response. Again, assuming that the valence of these emotional reactions indicates which deck GAGE will choose from, the results show that when the VMPFC is damaged, GAGE will choose from the bad deck rather than the good deck. This is consistent with the performance of VMPFCdamaged patients on the Iowa gambling task described above. Rather than behaving in an efficient manner and promoting decisions based on long-term survival, the organism will behave in ways that appear impulsive and even irrational, as did Phineas Gage.

\section{Experiment 2}

The goal of Experiment 2 was to test GAGE's ability to simulate experimental results concerning the integration of physiological arousal and cognition in determining emotional states (Schachter $\&$ Singer, 1962). Schachter and Singer (1962) injected participants with epinephrine and then had them fill out questionnaires in the presence of a confederate. In the euphoria condition, the cohort was pleasant, whereas in the anger condition, the cohort was unpleasant. Results showed that the same dose of epinephrine led to different emotional experiences depending on the context: Participants showed pleasant emotional experiences in the euphoric condition and unpleasant emotional experiences in the anger condition. Given a constant state of sympathetic activation, for which no immediately appropriate explanation is available (i.e., amygdala input is ambiguous), participants can be manipulated into states of euphoria or anger depending on the current context. It is important to note that the claims made by the Schachter theory of emotion have been drawn into question (e.g., Reisenzein, 1983). However, the goal of Experiment 2 was to simulate the role of context in cognitive-affective integration using GAGE. We are not concerned with the high-level mechanisms underlying the cognitive appraisal of one's emotional state. Instead, we are only interested in the mechanism whereby context exerts a moderating effect on the emotional reaction to different stimuli.

\section{Method}

Stimuli. On each trial in the experiment, GAGE was presented with a set of stimulus patterns consisting of activation vectors feeding into the VMPFC, the ventral tegmental area, the hippocampus, and the amygdala. Each pattern consisted of 35 active units (thereby exciting $35 \%$ of the neurons in the receiving region). To perform the tests, the VMPFC and the hippocampus required two separate activation patterns each. Note that in Experiment 1 context (i.e., hippocampal input) was held constant while body signal (i.e., amygdala input) was manipulated; whereas in Experiment 2 , context was manipulated and bodily input was held constant. The VMPFC received either an activation pattern representing the euphoria appraisal or an activation pattern representing the anger appraisal. The hippocampus received an activation pattern representing a pleasant context or an activation pattern representing an unpleasant context. Again, for simplicity, each set of patterns (euphoria-anger appraisal and pleasantunpleasant context) formed complimentary pairs.

Procedure. Initially, GAGE was trained on two combinations of stimuli: euphoria appraisal with pleasant context and anger appraisal with unpleasant context. This produced the requisite associations between VMPFC and hippocampal synapses in NAcc dendrites. Training occurred in an interleaved fashion over 4,000 time steps, with alternating stimulus combinations every 400 time steps. Hebbian learning was then turned off, and the model was presented with the euphoria-pleasant and angerunpleasant stimuli for epochs of 2,000 time steps each to establish baseline ensemble activity in the NAcc for the expected representations.

Finally, the model was presented with each of the test-stimulus combinations for epochs of 2,000 time steps. Test stimuli consisted of both appraisals (euphoria and anger) presented simultaneously with a given context (pleasant or unpleasant). This allowed us to test whether the network would elicit emotional reactions based on the current context. Amygdala and ventral tegmental area inputs were constant throughout training, baseline, and test.

Data collection and analysis. Data collection and analyses were as described in Experiment 1. 


\section{Results and Discussion}

To obtain a measure of GAGE's overall performance, we averaged all results over 50 replications of the experiment (with activation patterns and weights generated randomly for each replication). The results are presented in Figure 7.

The task of the network was to elicit a euphoric or anger appraisal given a context (e.g., pleasant). The network was tested by presenting the VMPFC and the hippocampus with activation patterns that simulated a condition in which both euphoric and anger appraisals were active in the VMPFC. The goal was to determine whether GAGE would elicit emotional reactions based on the current context.

As expected, hippocampal-determined context drove GAGE's behavior when the NAcc was presented with two different VMPFC representations simultaneously. Stored associations between the VMPFC and the hippocampus in NAcc dendrites are able to elicit a representation consistent with the current context.

\section{General Discussion}

Experiment 1 showed both how normal people perform cognitive-affective integration to succeed in the Iowa gambling task and why this integration breaks down in people with damage to the VMPFC. Consistent with A. Damasio's (1994) somaticmarker hypothesis, we have shown that the VMPFC and the amygdala interact to produce emotional signals indicating expected outcomes and that these expected outcomes compete with immediate outcomes for amygdala output. In addition, GAGE shows that temporal coordination between the VMPFC and the amygdala is a key component to eliciting emotional reactions to stimuli.

Experiment 2 showed how context is capable of exerting an effect on cognitive-affective integration. It does so by highlighting the importance of hippocampal input to the NAcc gateway. For the signals from the VMPFC and the amygdala to access brain areas responsible for higher order reasoning, context information from the hippocampus must unlock the NAcc gate, allowing this information to pass through.

In this article, we have presented a new computational model of decision making that is much more neurologically realistic than previous models. GAGE organizes neurons into populations related to brain areas, including the VMPFC, the hippocampus, and the amygdala. GAGE shows how the NAcc integrates cognitive information from the VMPFC and the hippocampus with emotional information from the amygdala. Consistent with A.

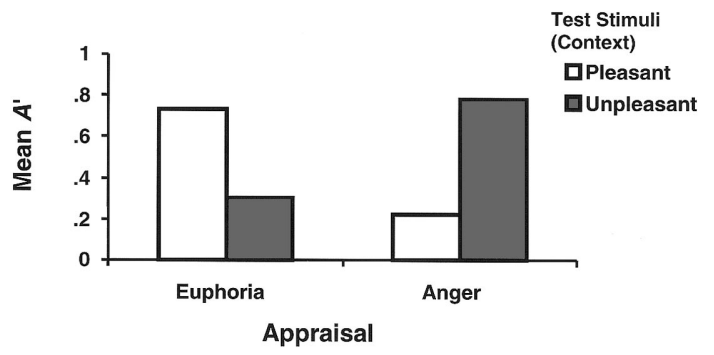

Figure 7. Mean $A^{\prime}$ for test stimuli as a function of appraisal with constant amygdala input.
Damasio's (1994) somatic-marker hypothesis, we have shown that the VMPFC and the amygdala interact to produce emotional signals indicating expected outcomes and that these expected outcomes compete with immediate outcomes for amygdala output. GAGE then moves beyond this claim, highlighting the importance of the NAcc gateway. For the signals from the VMPFC and the amygdala to pass through the NAcc to access brain areas responsible for higher order reasoning, the hippocampus must unlock the NAcc gate. Furthermore, the individual neurons in our model are more realistic than those used in most artificial neural network models because they exhibit the spiking behavior found in real neurons. This level of representation highlights another important aspect of cognitive-affective integration in the brain: time. GAGE shows that temporal coordination between the VMPFC and the amygdala is a key component to eliciting emotional reactions to stimuli.

GAGE is capable of producing effective decision-making strategies similar to those observed in humans with intact brains as well as the defective decision-making exhibited by modern-day equivalents of Phineas Gage. It does so by exhibiting cognitiveaffective integration during covert decision making. The central idea is that the VMPFC establishes predicted outcomes of responses through its connections with the amygdala and that this information is passed through the context-moderated gateway in the NAcc to promote behaviors that are most beneficial to the long-term survival of the organism within the current environment.

The Iowa gambling task is a clinical test of VMPFC damage, and GAGE is capable of simulating successful performance by people with normal cognitive-affective integration and unsuccessful performance by people with damage to the VMPFC. Also, by implementing the proposed neurological theory of how cognitive information and emotional information are integrated in effective decision making, GAGE has the capacity to move beyond this particular situation. In the Iowa gambling task, poor choices (picking the bad deck) are consistent with the current context. However, GAGE is more robust in its ability to model the neural basis for the production of emotional signals in decision making to promote achievement and long-term survival within a given environment. As was mentioned above, VMPFC damage causes the ability to reason-particularly within a social context - to become seriously impaired. Although the Iowa gambling task is an effective test of VMPFC damage, it fails to factor in the role of context. Thus, GAGE is capable of incorporating the role of context via its implementation of NAcc, ventral tegmental area, and hippocampal interrelations.

Experiment 2 provides a test of this capacity. Hippocampally determined context drives GAGE's behavior when the NAcc is presented with two different VMPFC representations simultaneously. Stored associations between the VMPFC and the hippocampus in NAcc dendrites are able to elicit a representation consistent with the current context.

The ability to replicate human performance in the Iowa gambling task and the capacity to incorporate the role of context demonstrate that GAGE, and the underlying neurological theory contribute to current understanding of decision making. According to the affect-as-information hypothesis, feelings influence decision making when experienced as reactions to the imminent decision, not if attributed to an irrelevant source (Clore, Schwartz, \& Conway, 1994). The constraints imposed by our neurological theory 
demand that for emotional information from the amygdala to be tied in with VMPFC activity as it passes through the NAcc gateway, the two must occur in close temporal proximity. That is, to bind cognition and emotion in the NAcc, VMPFC and amygdala activity must exhibit a coincidental increase in firing rate. Given this constraint, it does not seem surprising that researchers have found that feelings affect judgments when experienced as reactions to the imminent decision, not if attributed to an irrelevant source (Clore et al., 1994). If a given event (choosing a card) is associated with a given emotion, the two will co-occur in the brain-that is, they will exhibit a coincidental increase in firing rate. If this is the case, then they will impact the NAcc in close temporal proximity and be associated with one another. If, however, they are not associated with one another, they will fire out of phase with one another. As a result, they will not be bound in the NAcc, and the emotion will not be attributed to the event.

According to the risk-as-feelings hypothesis, feelings of fear in the face of a decision have an all-or-none characteristic (Loewenstein et al., 2001). Concretely, participants are sensitive to the possibility rather than the probability of negative outcomes. Here too, GAGE can shed some light on how the neural underpinnings of cognitive-affective integration produce this phenomenon. The basic idea is that feelings of fear, or any emotional reaction in a decision-making task, are insensitive to probability variations. Recall that NAcc neurons are being constantly bombarded with VMPFC- and amygdala-driven excitatory postsynaptic potentials. That is, VMPFC elicits several possible reactions to and/or appraisals of the current situation, and the hippocampus sorts through these options to find those that are context consistent. Implicit in this statement is the idea that VMPFC signals do not carry more or less weight relative to one another (i.e., they do not carry probability information). The emotional reactions elicited by the NAcc are immediate affective responses, providing fast but crude assessments of the current situation (A. Damasio, 1994; see also LeDoux, 1996). As such, the emotional reactions described here simply signal affective significance of a given reaction and/or appraisal. The role of probability might be factored into the decision process later, once the emotional reactions elicited here have been passed on to brain areas responsible for higher order decision-making processes. But, by then the emotion has already been elicited, hence the all-or-none characteristic of emotional reactions in decision making.

\section{Limitations of GAGE}

Although GAGE and the underlying neurological theory increase researchers' understanding of how cognitive information and emotional information are integrated in the NAcc during effective decision making, it is important to note that they do not answer every question. There are several levels on which GAGE is too simple or underspecified to address important questions.

Given the discussion presented thus far, one would have to conclude that if the NAcc were lesioned, the entire network would fall apart. However, given the plasticity of the brain, there are alternate brain areas (e.g., the cingulate or the supplementary motor area) that may compensate in the event of striate lesions. Given the limited number of brain areas currently implemented, GAGE is unable to address this issue. Also, the current implementations of GAGE focus on VMPFC lesions and the role of the hippocampus under uncertainty. Further simulations are needed to clarify what happens when either the amygdala or the hippocampus is lesioned. Finally, it is important to note that GAGE models only how the brain produces covert emotional reactions-it is not concerned with the high-level mechanisms underlying overt decisions or the cognitive appraisal of one's emotional state.

\section{Conclusion}

There is increasing appreciation in cognitive science that emotions are an integral part of decision making. GAGE and the underlying neurological theory increase researchers' understanding of the neural cause of the covert emotional reactions that are an essential part of effective decision making. In addition, GAGE's ability to incorporate the role of context in determining emotional signals endorses the ability to model other appraisal processes and other kinds of cognitive-affective integration in general.

Although there are limitations to the model, with time and closer implementation of neuroanatomical mechanisms, GAGE may be improved to simulate the process of human decision making to an even greater extent. As such, GAGE represents a first step in using computational neuroscience to aid in psychologists' understanding of human decision making and what goes wrong in the brain when these skills become impaired, and it may even help researchers to formulate better hypotheses concerning how to compensate for or correct the problems.

\section{References}

Aggleton, J. (2000). The amygdala: A functional analysis. Oxford, England: Oxford University Press.

Bechara, A., Damasio, A., Damasio, H., \& Anderson, S. (1994). Insensitivity to future consequences following damage to human prefrontal cortex. Cognition, 50, 7-15.

Bechara, A., Damasio, H., Tranel, D., \& Damasio, A. (1997, February 28). Deciding advantageously before knowing the advantageous strategy. Science, 275, 1293-1295.

Berns, G., McClure, S., Pagnoni, G., \& Montague, P. (2001). Predictability modulates human brain response to reward. The Journal of Neuroscience, 21, 2793-2798.

Breiter, H., Aharon, I., Kahneman, D., Dale, A., \& Shizgal, P. (2001). Functional imaging of neural responses to expectancy and experience of monetary gains and losses. Neuron, 30, 619-639.

Calabresi, P., De Murtas, M., \& Bernardi, G. (1997). The neostriatum beyond the motor function: Experimental and clinical evidence. Neuroscience, 78, 39-60.

Churchland, P. (1996). Feeling reasons. In A. R. Damasio, H. Damasio, \& Y. Christen (Eds.), Neurobiology of decision-making (pp. 181-199). Berlin, Germany: Springer.

Clore, G., Schwartz, N., \& Conway, M. (1994). Affective causes and consequences of social information processing. In R. Wyer \& T. Srull (Eds.), Handbook of social cognition (Vol. 1, pp. 323-417). Hillsdale, NJ: Erlbaum.

Damasio, A. (1994). Descartes' error: Emotion, reason and the human brain. New York: Avon Books.

Damasio, H., Grabowski, T., Frank, R., Galburda, A. M., \& Damasio, A. R. (1994, May 20). The return of Phineas Gage: Clues about the brain from the skull of a famous patient. Science, 264, 1102-1104.

Everitt, B., Landau, S., \& Leese, M. (2001). Cluster analysis (4th ed.). New York: Oxford University Press.

Finucane, M. L., Alhakami, A. S., Slovic, P., \& Johnson, S. M. (2000). The 
affect heuristic in judgments of risks and benefit. Behavioral Decision Making, 13, 1-17.

Gerstner, W. (1999). Spiking neurons. In W. Maass \& C. Bishop (Eds.), Pulsed neural networks (pp. 3-54). Cambridge, MA: MIT Press.

Gerstner, W., \& van Hemmen, J. (1994). Coding and information processing in neural networks. In E. Domany, J. van Hemmen, \& K. Schulten (Eds.), Models of neural networks II (pp. 1-93). New York: SpringerVerlag.

Grace, A., \& Moore, H. (1998). Regulation of information flow in the nucleus accumbens: A model for the pathophysiology of schizophrenia. In M. Lenzenweger \& R. Dworkin (Eds.), Origins and development of schizophrenia: Advances in experimental psychopathology (pp. 123157). Washington, DC: American Psychological Association.

Harlow, J. M. (1848). Passage of an iron rod through the head. Boston Medical and Surgical Journal, 39, 389-393.

Hitchcott, P., \& Phillips, G. (1997). Amygdala and hippocampus control dissociable aspects of drug associated conditioned rewards. Psychopharmacology, 131, 187-195.

Kempter, R., Gerstner, W., \& van Hemmen, J. (1999). Hebbian learning and spiking neurons. Physical Review E: Statistical, Nonlinear, and Soft Matter Physics, 59, 4498-4514.

Knutson, B., Adams, C., Fong, G., \& Hommer, D. (2001). Anticipation of increasing monetary reward selectively recruits nucleus accumbens [Electronic version]. The Journal of Neuroscience, 21, RC159. Retrieved from http://www.jneurosci.org/cgi/reprint/21/16/RC159.pdf

Knutson, B., Westdorp, A., Kaiser, E., \& Hommer, D. (2000). fMRI visualization of brain activity during a monetary incentive delay task. NeuroImage, 12, 20-27.

LeDoux, J. (1996). The emotional brain. New York: Simon \& Schuster.

Lerner, J. S., \& Keltner, D. (2000). Beyond valence: Toward a model of emotion-specific influences on judgment and choice. Cognition \& Emotion, 14, 473-493.

Lerner, J. S., \& Keltner, D. (2001). Fear, anger, and risk. Journal of Personality and Social Psychology, 81, 146-159.
Loewenstein, G. F., Weber, E. U., Hsee, C. K., \& Welch, N. (2001). Risk as feelings. Psychological Bulletin, 127, 267-286.

Macmillan, M. (2000). An odd kind of fame: Stories of Phineas Gage. Cambridge, MA: MIT Press.

Mischel, W., \& Shoda, Y. (1995). A cognitive-affective system theory of personality: Reconceptualizing situations, dispositions, dynamics, and the invariance in personality structure. Psychological Review, 102, 246268 .

Mogenson, G., Jones, D., \& Yim, C. (1980). From motivation to action: Functional interface between the limbic system and the motor system. Progress in Neurobiology, 14, 69-97.

Nerb, J., \& Spada, H. (2001). Evaluation of environmental problems: A coherence model of cognition and emotion. Cognition \& Emotion, 15, $521-551$.

O'Donnell, P. (1999). Ensemble coding in the nucleus accumbens. Psychobiology, 27, 187-197.

O'Donnell, P., \& Grace, A. (1995). Synaptic interactions among excitatory afferents to nucleus accumbens neurons: Hippocampal gating of prefrontal cortical input. The Journal of Neuroscience, 15, 3622-3639.

Reisenzein, R. (1983). The Schachter theory of emotion: Two decades later. Psychological Review, 94, 239-264.

Rottenstreich, Y., \& Hsee, C. K. (2001). Money, kisses, and electric shocks: On the affective psychology of risk. Psychological Science, 12, 185-190.

Schachter, S., \& Singer, J. (1962). Cognitive, social and physiological determinants of emotional state. Psychological Review, 69, 379-399.

Snodgrass, J., Levy-Berger, G., \& Hayden, M. (1985). Human experimental psychology. New York: Oxford University Press.

Thagard, P. (2000). Coherence in thought and action. Cambridge, MA: MIT Press.

Thagard, P. (2003). Why wasn't O. J. convicted? Emotional coherence in legal inference. Cognition \& Emotion, 17, 361-383.

Wagar, B., \& Thagard, P. (2003). Using computational neuroscience to investigate the neural correlates of cognitive-affective integration during covert decision making. Brain and Cognition, 53, 398-402. 


\section{Appendix}

\section{Spiking Neuron Model}

For a full description of the following equations, see also Wagar and Thagard (2003).

We used a slightly modified leaky integrate-and-fire neuron, based heavily on Gerstner's (1999) spike response model (Gerstner \& van Hemmen, 1994). The total membrane potential $\alpha_{i}$ of a given neuron $i$ at time $t$ is given by

$$
\alpha_{i}=\sum_{t_{i}^{f} \in \mathrm{F}_{i}} \eta_{i}\left(t-t_{i}^{f}\right)+\sum_{j \in \Gamma_{i} t_{j}^{f} \in F_{j}} \omega_{i j} \varepsilon_{i j}\left(t-t_{j}^{f}\right) .
$$

The right hand side of Equation A1 can be decomposed into two response functions. The left term on the right side of Equation A1 represents the response of neuron $i$ to the set $F_{i}$ of all its own previous spike emissions $t_{i}^{f}$. In the event of a spike, a short-term, decaying negative contribution $\eta(s)$ is added to the total membrane potential given by

$$
\eta(s)=-\mu \theta_{i} \exp \left(\frac{-s}{\tau}\right) H(s),
$$

where $s$ is the time $\left(t-t_{i}^{f}\right)$ since the last spike of neuron $i, \theta$ is the firing threshold of neuron $i$, and $\mu$ and $\tau$ are constants that scale the amplitude and the decay rate of $\eta(s)$, respectively. Finally, $H(s)$ is the Heaviside step function defined by

$$
H(s)= \begin{cases}0 & \text { for } s<0 \\ 1 & \text { otherwise }\end{cases}
$$

The last term on the right of Equation A1 represents the response of neuron $i$ to the set $F_{j}$ of all previous spike emissions $t_{j}^{f}$ for the set of all afferent connections $\Gamma_{j}$ to neuron $i$. The variable $\omega_{i j}$ represents the connection strength between the postsynaptic neuron $i$ and a given presynaptic neuron $j$. The postsynaptic potential induced in neuron $i$ at time $t$ in response to the firing of presynaptic neuron $j$ at time $t_{i}^{f}$ is given by

$$
\varepsilon(s)=\delta\left[\left(\frac{s}{\tau_{s}^{2}}\right) \exp \left(\frac{-s}{\tau_{s}}\right)\right] H(s),
$$

where $\delta$ is a constant representing the valence of the postsynaptic potential ( 1 if excitatory, -1 if inhibitory), $s$ is the time $\left(t-t_{j}^{f}\right)$ since the last spike of neuron $j$, and $\tau_{s}$ is a time constant representing the rise time of the postsynaptic potential. Again, $\mathrm{H}(s)$ is the Heaviside step function described in Equation A3.

At each time step the connection strengths, $\omega_{i j}$, are updated synchronously according to a Hebbian learning rule, modeled roughly after Kempter, Gerstner, and van Hemmen (1999). In our model, the connection strength, $\omega_{i}$, between the postsynaptic neuron, $i$, and a given presynaptic neuron, $j$, is adjusted according to the delay between the time, $t_{j}^{f}$, of the most recent presynaptic spike arrival and the time, $t_{i}^{f}$, of the most recent postsynaptic firing. The formulation of the change of connection strength, $\omega_{i j}$, is given by

$$
\begin{aligned}
\Delta \omega_{i j}=\gamma_{i j}\left\{\exp \left(\frac{s}{\tau_{\text {syn }}}\right)\left[A+\left(1-\frac{s}{\bar{\tau}+}\right)+A-\left(1-\frac{s}{\bar{\tau}-}\right)\right]\right\}, & \text { for } s<0 ; \gamma_{i j} \text {, for } s=0 ;
\end{aligned}
$$

and

$$
\gamma_{i j}\left[A+\exp \left(\frac{-s}{\tau+}\right)+A-\exp \left(\frac{-s}{\tau-}\right)\right], \text { for } s>0
$$

where $s$ is the delay $\left(t_{i}^{f}-t_{i}^{f}\right)$ between presynaptic spike arrival and postsynaptic firing; $\tau_{\text {syn }}, \bar{\tau}+, \bar{\tau}-, \tau+$, and $\tau-$ are time constants; and $A+$ and $A-$ are constants that scale the strength of synaptic potentiation and depression, respectively. Finally, $\lambda_{i j}$ is the learning rate for the synapse connecting the presynaptic neuron $j$ to the postsynaptic neuron $i$.

Received July 31, 2002

Revision received January 4, 2003

Accepted February 3, 2003

\section{Wanted: Your Old Issues!}

As APA continues its efforts to digitize journal issues for the PsycARTICLES database, we are finding that older issues are increasingly unavailable in our inventory. We are turning to our long-time subscribers for assistance. If you would like to donate any back issues toward this effort (preceding 1982), please get in touch with us at journals@apa.org and specify the journal titles, volumes, and issue numbers that you would like us to take off your hands. 